\title{
DESCRIPCIÓN DE LA LARVA DE PHYLLOPHAGA LENIS (COLEOPTERA: MELOLONTHIDAE) EN SANTA CRUZ XOXOCOTLÁN, OAXACA, MÉXICO, CON NOTAS SOBRE SU BIOLOGÍA
}

\section{Julián HERNÁNDEZ-CRUZ, ${ }^{1}$ Miguel Ángel MORÓN, ${ }^{2}$ JAIMe RUIZ-VEGA, ${ }^{1}$ José ANTonio SÁNCHEZ- GARCÍA, ${ }^{1}$ LAURA MARTÍNEZ-MARTÍNEZ ${ }^{1}$ y RAFAEL PÉREZ PACHECO ${ }^{1}$}

\author{
${ }^{1}$ Instituto Politécnico Nacional. CIIDIR Unidad Oaxaca. Hornos 1003, Col. Nochebuena. Sta. Cruz Xoxocotlán, \\ Oaxaca 71230, México.<jhcciidir@yahoo.com.mx> \\ ${ }^{2}$ Instituto de Ecología A.C. Carretera Antigua a Coatepec 351, El Haya, Xalapa, Veracruz. 91070, México \\ $<$ miguel.moron@inecol.mx>
}

Recibido: 12/05/2015; aceptado: 09/11/2015

\begin{abstract}
Hernández-Cruz, J., Morón, M. Á., Ruiz-Vega, J., Sánchez-García, J. A., Martínez-Martínez, L. \& Pérez Pacheco, R. 2016. Descripción de la larva de Phyllophaga lenis (Coleoptera: Melolonthidae) en Santa Cruz Xoxocotlán, Oaxaca, México, con notas sobre su biología. Acta Zoológica Mexicana (n. s.), 32(1): 55-61.
\end{abstract}

RESUMEN. Se describe el tercer estadio larval de Phyllophaga lenis (Horn, 1887) con especímenes criados en laboratorio, cuyos progenitores fueron capturados en un campo agrícola de Santa Cruz Xoxocotlán, Oaxaca. Se incluyen las imágenes de los caracteres diagnósticos, notas sobre su biología y hábitos, comentarios acerca de las diferencias con otras especies mexicanas parecidas y una clave para separar las larvas de 15 especies de Phyllophaga en México.

Palabras clave: escarabajos de junio, biología, estados inmaduros, plagas agrícolas, gallina ciega, clave taxonómica.

\section{INTRODUCCIÓN}

De acuerdo con Aragón et al. (2005) sólo se ha descrito el ciclo vital completo de 24 especies de Phyllophaga de las 820 enlistadas para América y sólo se han descrito las larvas de 14 especies de las casi 400 citadas en México (Aragón \& Morón, 2004a). Ante este escenario, es necesario promover la investigación relativa a la descripción morfológica detallada de las larvas de Phyllophaga del territorio nacional y documentar los resultados obtenidos, con el propósito de incrementar la información al respecto. Este conocimiento básico es necesario cuando se implementa un programa de control para las especies que se constituyen en plagas subterráneas de cultivos. Esta publicación tiene como objetivo describir por primera vez la larva de tercer estadio de Phyllophaga lenis (Horn, 1887) cuya elevada abundancia y descripción detallada del imago fueron documentadas por Hernández-Cruz et al. (2014) en Santa Cruz Xoxocotlán, Oaxaca.
Hernández-Cruz, J., Morón, M. Á., Ruiz-Vega, J., SánchezGarcía, J. A., Martínez-Martínez, L. \& Pérez Pacheco, R. 2016. Description of the larva of Phyllophaga lenis (Coleoptera: Melolonthidae, Melolonthinae) from Santa Cruz Xoxocotlan, Oaxaca, Mexico, with notes on its biology. Acta Zoológica Mexicana (n. s.), 32(1): 55-61.

ABSTRACT. The third instar larvae of Phyllophaga lenis (Horn, 1887 ) is described with specimens reared in laboratory whose progenitors were captured in agriculture field of Santa Cruz Xoxocotlán, Oaxaca. The pictures of diagnostic characters, notes on it biology and habits, comments about the differences with similar species, and a key to identify larvae of fifteen Mexican species of Phyllophaga are included.

Key words: June beetles, biology, immature stages, agricultural pests, white grub, taxonomic key.

Características del área de estudio. La mayor parte de los trabajos de campo se realizaron en Santa Cruz Xoxocotlán (Fig. 1), cuya altitud promedio es de $1560 \mathrm{~m}$, temperatura promedio de $20.4^{\circ} \mathrm{C}$, mínima y máxima extremas de 9.1 y $32{ }^{\circ} \mathrm{C}$, y una precipitación anual que osciló entre 649 y $795 \mathrm{~mm}$ en los últimos diez años. Predomina un clima subhúmedo, semicálido (BS1h). Existe poca vegetación nativa debido al manejo que los campesinos hacen de sus cultivos. Actualmente la vegetación dominante es de tipo xerófita, asociada al chaparral, principalmente guamúchil [Pithecellobium dulce (Roxb.) Benth.], mezquites [Prosopis juliflora (Swartz) DC.], huizache [Acacia farnesiana (L.) Willd.], cactáceas, agaves y pastos. Las áreas arboladas (principalmente con pinos y encinos) o cubiertas con carrizo (Phragmites communis Trin.) son cada vez más escasas. Los tipos de suelo son vertisol pélico y en menores proporciones acrisol férrico, luvisol crómico y luvisol pélico. El uso del suelo en el área de estudio está destinado básicamente a dos actividades: la agricultura de 


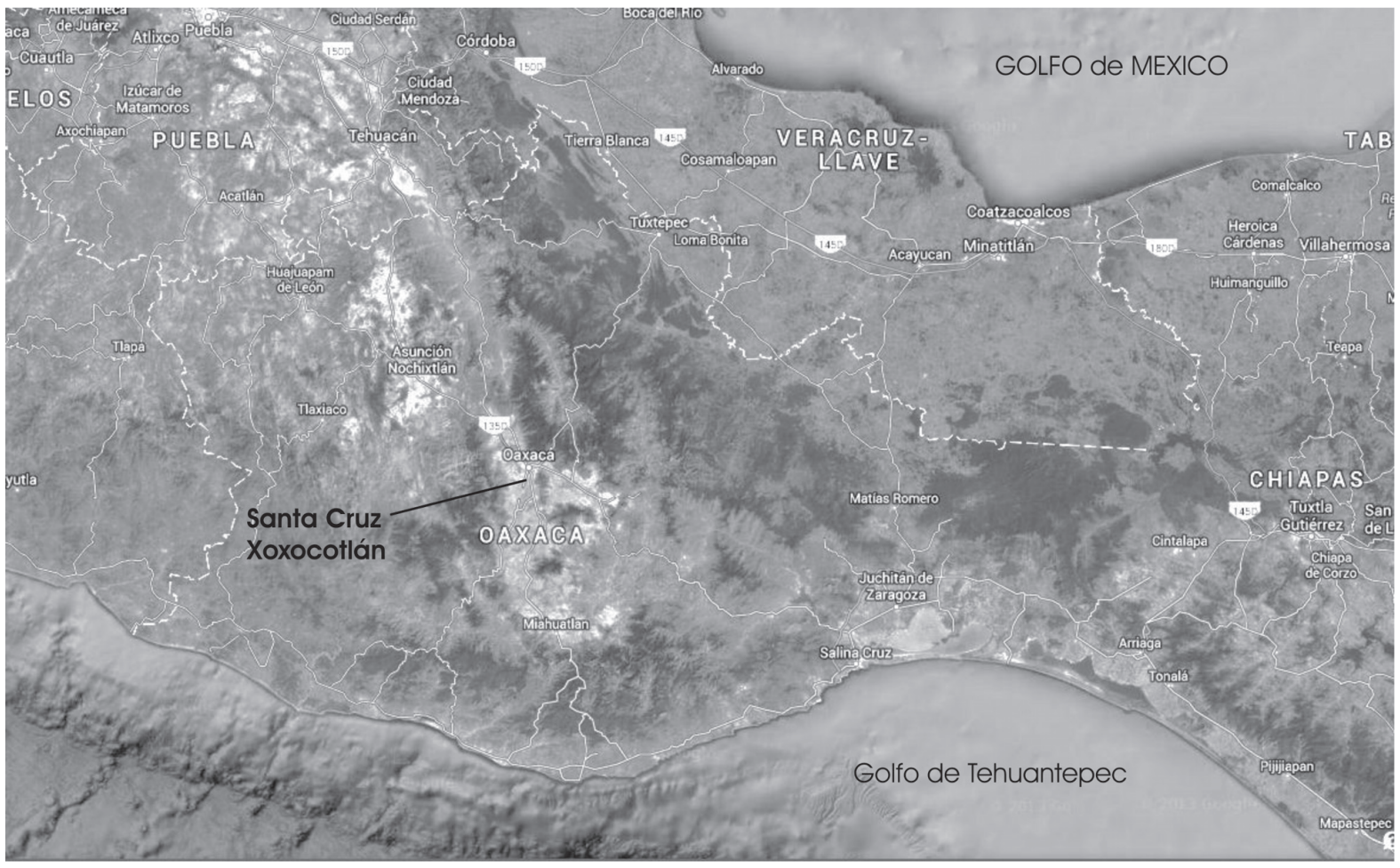

Figura 1. Ubicación de Santa Cruz Xoxocotlán, Oaxaca. Imagen modificada de Google Maps.

temporal/riego y el pastoreo de ovi-caprinos en menor escala (Velasco \& Aguilar 2000).

\section{MATERIALES Y MÉTODOS}

Cría de larvas. El método para la cría de larvas que se siguió en este trabajo se basó en el descrito por Aragón y Morón (2004b) que se comenta a continuación: se inició con la captura de adultos en cópula o hembras de $P$. lenis, que se colocaron individualmente o por parejas en vasos de plástico de $250 \mathrm{ml}$, provistos con suelo y follaje del lugar donde se capturaron, se taparon con tela organza y papel aluminio con perforaciones para promover la ventilación y evitar fugas; posteriormente se llevaron al laboratorio del CIIDIR-IPN-Oaxaca y se mantuvieron a temperatura ambiente. Después de dos días se separaron los huevos, los adultos que estaban vivos se regresaron al recipiente y se humedeció el suelo. Esta actividad se realizó cada dos días hasta que murieron los adultos, que se montaron en alfileres entomológicos para confirmar su identidad. Los huevos obtenidos se depositaron en vasos de plástico con $100 \mathrm{~g}$ de suelo esterilizado rico en materia orgánica. Se dio seguimiento a las larvas que eclosiona- ron y se cambió el suelo cada 15 días, agregando plántulas de maíz.

Las larvas de $P$. lenis, criadas en el laboratorio y seleccionadas para su descripción morfológica se fijaron y conservaron previamente siguiendo la metodología propuesta por Morón y Terrón (1988). El estudio morfológico se fundamentó en seis larvas de tercer estadio (Fig. 2) criadas en el laboratorio para hacer la descripción e ilustraciones y obtener las dimensiones de la cápsula cefálica y el cuerpo, así como para evaluar la quetotaxia diagnóstica con la ayuda de un estereomicroscopio Carl Zeiss equipado con una cámara digital Nikon y micrómetro ocular. La edición de imágenes se hizo con el programa Adobe Photoshop Versión CS5.1. Los caracteres y la terminología utilizada son los propuestos por Böving (1942) y Morón (1986). Muestras de los ejemplares estudiados se conservan en las colecciones entomológicas del CIIDIR-IPN-Oaxaca y del Instituto de Ecología, A. C. Xalapa (IEXA).

\section{RESULTADOS}

Phyllophaga lenis (Horn, 1887) Tercer estadio larvario (Figs. 2-8). 

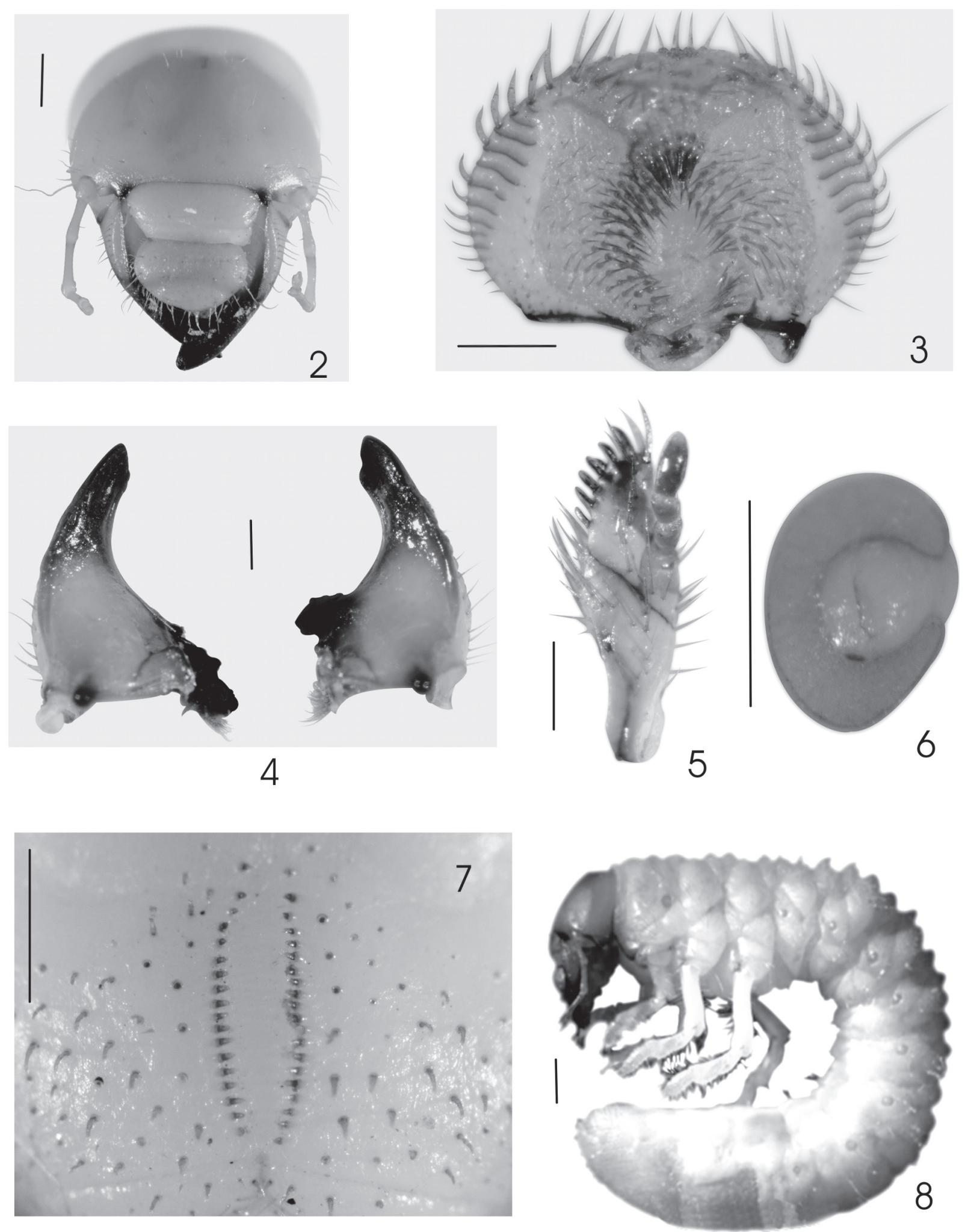

Figuras 2-8. Phyllophaga lenis. 2) Aspecto frontal de la cápsula cefálica de la larva. 3) Epifaringe. 4) Vista ventral de las mandíbulas izquierda y derecha. 5) Aspecto dorsal de la maxila derecha. 6) Placa respiratoria abdominal. 7) Raster. 8) Larva de tercer estadio. Líneas de escala $=0.5$ $\mathrm{mm}$ excepto en figuras 2 y $8=1 \mathrm{~mm}$. 
Descripción. Cabeza: Anchura promedio de cápsula cefálica $4.7 \mathrm{~mm}(N=6)$. Superficie del cranium casi lisa, parda amarillenta (Fig. 3). Frente con una seda frontal exterior y una seda frontal posterior a cada lado, 12 sedas frontales anteriores y una seda larga en cada ángulo anterior de la frente; el resto de la superficie craneal con cinco sedas dorso-epicraneales, una seda epicraneal y 16 sedas para-antenales a cada lado. Clípeo con dos sedas centrales y dos sedas laterales a cada lado. Labro ligeramente asimétrico con los márgenes laterales redondeados, con 11 sedas posteriores, dos sedas centrales y una seda lateral y una seda preapical a cada lado. Manchas oculares ausentes. Epifaringe (Fig. 4) sin zygum y con epizygum alargado, irregular, pardo rojizo, haptomerum prominente con 18-19 heli dispuestos en tres hileras transversales irregulares, cada plegmatium formada por 11 plegmata anchos. Proplegmatia ausente. Dexiophoba escasa, irregular. Laeophoba abundante y continua, extendida a través del extremo mesial de la laeotorma. Dexiotorma alargado con el extremo mesial ligeramente aguzado. Laeotorma corta y ancha; apotorma y epitorma indefinidas; pternotorma ancha con el extremo aguzado. Haptolachus desnudo. Placa esclerosada ancha y casi cubierta por la dexiophoba. Cono sensorial no identificado. Crepis ligeramente marcado. Cada acanthoparia con 12-13 sedas espiniformes ligeramente recurvadas. Chaetoparia con sensilas abundantes en el límite con la gymnoparia.

Mandíbulas (Fig. 5) sin área estriduladora ventral. Áreas incisivas de ambas mandíbulas con una lámina cortante distal separada de un dientecillo incisivo proximal por una escotadura incisiva, márgenes internos sin dientes. Área molar de la mandíbula izquierda con un lóbulo distal (MI) bien desarrollado, acia reducido, brustia multisetosa. Área molar de la mandíbula derecha poco desarrollada, calx prominente, brustia multisetosa. Maxilas (Fig. 6) con un uncus terminal largo en la galea y tres unci largos subapicales alineados en la lacinia y con hileras de cuatro a cinco heli a cada lado. Área estriduladora maxilar con 12 dientecillos agudos. Escleroma hipofaringeo asimétrico, prominente, redondeado. Último artejo antenal con un área sensorial ovalada en el dorso y dos áreas sensoriales más pequeñas en la superficie ventral.

Tórax: Las placas respiratorias (Fig. 7) miden 0.43 $\mathrm{mm}$ de ancho y $0.34 \mathrm{~mm}$ de largo, amarillentas, uniformemente recurvados en forma de "C", la distancia entre los lóbulos es menor al diámetro dorso ventral de la bulla ovalada y poco prominente. Pronoto con un escleroma lateral irregular a cada lado, poco contrastante con sedas medianas esparcidas. Escudos torácicos con escasas hileras transversales de sedas largas y medianas esparcidas, pero sin sedas espiniformes. Protarsos y mesotarsos con las uñas similares, alargadas, agudas, con dos sedas prebasales, uñas metatarsales reducidas, mamilares, con dos sedas largas.

Abdomen: Las placas respiratorias de los segmentos abdominales tienen diferentes tamaños, en el $1^{\circ}$ mide 0.27 $\mathrm{mm}$ de ancho y $0.20 \mathrm{~mm}$ de largo, del $2^{\circ}$ al $4^{\circ}$ miden 0.3 $\mathrm{mm}$ de ancho y $0.2 \mathrm{~mm}$ largo; en el $5^{\circ}$ y $6^{\circ}$ alcanzan 0.32 $\mathrm{mm}$ de ancho y $0.28 \mathrm{~mm}$ de largo; en el $7^{\circ}$ alcanza 0.38 $\mathrm{mm}$ de ancho y $0.33 \mathrm{~mm}$ de largo; y en el $8^{\circ}$ segmento mide $0.24 \mathrm{~mm}$ de ancho y $0.23 \mathrm{~mm}$ de largo. Las placas respiratorias son amarillentas regularmente recurvadas en forma de "C", a excepción del primero, la distancia entre los lóbulos de la placa respiratoria es menor al diámetro dorso-ventral de la bulla que de igual manera, excepción del primero, es ovalada y poco prominente. Dorsa de los segmentos abdominales $1^{\circ}$ a $6^{\circ}$ con numerosas sedas espiniformes y algunas hileras de sedas largas distribuidas en sentido transversal. Segmentos abdominales $7^{\circ}$ a $9^{\circ} \sin$ sedas espiniformes, con ocho a 11 sedas largas dispuestas en hileras transversales. Venter de los segmentos abdominales $1^{\circ}$ a $9^{\circ}$ con hileras de 10 a 14 sedas largas. Raster (Fig. 8) con un par de palidia longitudinales ligeramente convergentes en ambos extremos con $1.6 \mathrm{~mm}$ de longitud, separadas $0.43 \mathrm{~mm}$ en la parte más ensanchada de la séptula; cada palidium está formado por 19-21 pali cortos ligeramente recurvados, con ápices agudos dirigidos hacia la línea media. Tegilla con 39 a 40 sedas hamate comprimidas y recurvadas hacia su ápice. Campus con seis sedas largas y finas. Barbula abundante. Labio anal inferior estrecho y profundamente hendido en su línea media. Abertura anal en forma de " $v$ " muy abierta (Fig. 8). Longitud total por el dorso del cuerpo: $46 \mathrm{~mm}$.

Material examinado. Seis larvas de tercer estadio criadas a partir de parejas colectadas en MEXICO: Oaxaca, Santa Cruz Xoxocotlán, 20-V-2010, J. Hernández-Cruz (CIIDIR-IPN, Oaxaca; IEXA).

Biología. El primer macho de la temporada 2010 fue atraído por la trampa de luz el 17 de abril 2010 en Santa Cruz Xoxocotlan, días después de una fuerte lluvia. Los últimos ejemplares se colectaron el 26 de junio en Lomas de Nazareno Xoxocotlán. En el césped de un jardín los individuos empezaron a volar a las 20:15 horas (horario de verano).. A las 20:28 horas se posaron sobre la pantalla de la trampa, donde permanecieron hasta las 21:15 horas cuando el primer individuo voló y a las 22:40 horas todos abandonaron la pantalla. 
Las hembras se posaron sobre distintos plantas del jardín, como rosales, aguacateros, helechos, ciruelos y, en el campo, principalmente en huizache, donde atrajeron a los machos. La cópula inició entre 20:30 y 20:35 horas y finalizó entre 20:55 y 21:00 horas. Después de la cópula la hembra consume retoños tiernos de huizache durante unos 20 minutos antes de iniciar el vuelo para buscar un sitio donde enterrarse.

El estudio del ciclo vital se inició con un total de 94 huevos depositados por 28 hembras en un periodo aproximado de tres a cinco días posteriores a la colecta, de los cuales se obtuvieron 63 larvas de primer estadio, 49 de segundo y 39 larvas de tercer estadio. El periodo de incubación de los huevos fue de 9 a 16 días con un promedio de 12.75 días. El primer estadio larval se desarrolló en un periodo de 13 a 25 días con un promedio de 19.33 días; el segundo tuvo una duración de 32 a 49 días con un promedio 42.25 días. No fue posible observar el desarrollo completo del tercer estadio, pero debe durar cuando menos 90 días.

El confinamiento de hembras individualmente en terrarios para que depositaran sus huevos pareció inhibir dicha actividad, porque se obtuvo un promedio de 3.35 huevos por hembra. Sin embargo, cuando se reunieron varias hembras en un terrario el promedio de ovipostura aumentó significativamente a 7.96. También se observó que la mayoría de las larvas no consumieron las raíces de las plántulas de maíz adicionadas al terrario, especialmente las larvas de primer estadio, pero se observó que los granos de maíz colocados para que germinarán en ocasiones resultaron mordisqueados.

\section{DISCUSIÓN}

Phyllophaga lenis es la tercera larva del grupo "anodentata" (sensu Morón 1986) que se estudia con detalle desde que Morón (1986) y Aragón y Morón (2004a) publicaron la descripción pormenorizada de $P$. brevidens (Bates) y $P$. vetula respectivamente. Espinosa-Islas et al. (2005) documentaron ilustraciones de la epifaringe y el raster de $P$. vetula, sin incluir una descripción detallada de las mismas. Las diferencias más notorias observadas entre las larvas de $P$. lenis y $P$. vetula son: la cabeza es más ancha en $P$. vetula, los unci de la lacinia maxilar son más largos en $P$. lenis, el número de pali en cada palidia es menor en $P$. lenis y sus palidia son ligeramente convergentes en ambos extremos (Cuadro 1).

Las larvas de las tres especies antes mencionadas se distribuyen ampliamente en México, exceptuando las
Cuadro 1. Diferencias entre larvas de tercer estadio de $P$. lenis y $P$. vetula.

\begin{tabular}{lcc}
\hline Caracteres & P. lenis & P. vetula \\
\hline Longitud dorsal & $46 \mathrm{~mm}$ & $56 \mathrm{~mm}$ \\
Ancho de cápsula & $4.7 \mathrm{~mm}$ & $5.6-5.8 \mathrm{~mm}$ \\
cefálica & $15-17$ & $10-12$ \\
Sedas para-antenales & Ancho & Alargado \\
$\begin{array}{l}\text { Dexiotorma } \\
\text { Chaetoparia (sensilas) }\end{array}$ & Abundantes & Escasas \\
Unci de la lacinia & Largos & Cortos \\
$\begin{array}{l}\text { Dientecillos } \\
\text { estriduladotes de la } \\
\text { maxila }\end{array}$ & 12 & 11 \\
Lóbulo distal del área & Muy desarrollado & Desarrollado \\
molar (M1) & & \\
Palidia & Ligeramente & Casi paralelos \\
& convergentes en & \\
Cada palidium & Con 18-20 pali & Con 23-30 pali \\
\hline
\end{tabular}

penínsulas de Yucatán y Baja California (Morón 2003). Debido a su abundancia y frecuencia de captura $P$. lenis está incluida en el listado de especies potencialmente dañinas para los cultivos (Morón 2003), aunque en los Valles Centrales de Oaxaca no se tiene conocimiento de daños atribuidos a esta especie que sobrepasen el umbral económico. De acuerdo con las observaciones realizadas durante el presente trabajo, es factible que las larvas de esta especie tengan hábitos facultativos con preferencias saprófagas, y que solo bajo condiciones extremas pueda causar daños de consideración en las raíces de las plantas cultivadas.

Clave para separar las larvas de tercer estadio de 15 especies de Phyllophaga en México

(Modificada de Ramírez-Salinas et al. 2000 y Aragón \& Morón 2004a).

1. Epifaringe con proplegmatia .............2

1 'Epifaringe sin proplegmatia ..............5

2. Placa respiratoria del $7^{\circ}$ segmento abdominal con diámetro más grande que la placa del $6^{\circ}$ segmento. $P a$ lidia muy recurvadas, cercanamente convergentes en ambos extremos. Anchura de la cápsula cefálica 8 a 8.6 $\mathrm{mm}$............ (Phyllophaga) ilhuicaminai Morón $2^{\prime}$ Placa respiratoria del $7^{\circ}$ segmento abdominal con diámetro similar al de la placa del $6^{\circ}$ segmento. Palidia casi rectas o divergentes en ambos extremos. Anchura de la cápsula cefálica menor a $5.2 \mathrm{~mm}$.............. 3 3. Pali dirigidos hacia los lados. Palidia divergen- 
tes en los extremos formados por 14-20 pali largos y agudos. Anchura de la cápsula cefálica $4 \mathrm{~mm}$ ................ (Phyllophaga) pubicauda (Bates) 3' Pali dirigidos hacia la línea media . . . . . . . . . . 4 4. Extremos posteriores de las palidia ligeramente convergentes, cada palidium formado por 8-15 pali cortos. Anchura de la cápsula cefálica 3.8 a 4.1 $\mathrm{mm}$............... (Chlaenobia) tumulosa (Bates) 4' Palidia casi paralelos, cada palidium formado por 14-21 pali cortos. Anchura de la cápsula cefálica 4.5-5.1 . . . . . . P. (Phyllophaga) ravida (Blanchard) 5. Diámetro dorsoventral de las placas respiratorias de los segmentos abdominales $6^{\circ}$ y $7^{\circ}$ claramente más pequeño que las placas precedentes. Anchura de la cápsula cefálica $3.6 \mathrm{~mm}$... P. (Listrochelus) cuicateca Morón y Aragón 5 ' Diámetro dorsoventral de las placas respiratorias de los segmentos abdominales $6^{\circ}$ y $7^{\circ}$ semejantes a la placa del $5^{\circ}$ segmento...................... 6 6. Frente con 26-30 sedas anterofrontales y 30-54 sedas posterofrontales. Palidia ligeramente convergentes en ambos extremos, cada palidium formado por 27 a 37 pali cortos. Anchura de la cápsula cefálica 7.4-8.0 $\mathrm{mm}$.............. (Triodonyx) lalanza Saylor 6' Frente con 8-15 sedas anterofrontales y 2-4 sedas posterofrontales. Palidia convergentes o paralelas, cada palidium formado por menos de 30 pali. Anchura de la cápsula cefálica 4.1-6.7 mm . ............... 7 7. Palidia muy recurvadas. Septula ovalada, bien delimitada ......................... 8 7' Palidia ligeramente recurvadas o casi rectas. Septula alargada, poco delimitada en los extremos . . . . . . . . 9 8. Cada palidium con 19-25 pali cortos. Frente ligeramente rugosa. Anchura de la cápsula cefálica 4.9-5.2 $\mathrm{mm}$...... P. (Phyllophaga) testaceipennis (Blanchard) 8' Cada Palidium con 18-20 pali largos. Frente casi lisa. Anchura de la cápsula cefálica $3.0 \mathrm{~mm}$.... P. (Phytalus) macrocera (Bates)

9. Palidia casi paralelos, formados por 23-30 pali . . . 10

9' Palidia ligeramente convergentes en uno o ambos extremos, formados por 11-27 pali. . . . . . . . . . . 11 10. Septula lisa. Placa respiratoria del $7^{\circ}$ segmento abdominal con diámetro similar al de la placa del $6^{\circ}$ segmento. Maxilas con 11- 18 dientecillos estriduladores. Anchura de la cápsula cefálica 5.6-6.5 $\mathrm{mm}$........ P. (Phyllophaga) menetriesi (Blanchard) 10 ' Septula con estrías transversales. Placa respiratoria del $7^{\circ}$ segmento abdominal con diámetro un poco mayor al de la placa del $6^{\circ}$ segmento. Maxilas con 11 dientecillos estriduladores. Anchura de la cápsula cefálica 5.8 $\mathrm{mm} \ldots \ldots \ldots \ldots . . . .$. (Phyllophaga) vetula (Horn) 11. Frente casi lisa, con una seda posterofrontal a cada lado. Palidia ligeramente convergentes en ambos extremos, cada palidium formado por 15 a 27 pali . . . . . . . . 12 11 ' Frente finamente rugosa, con dos o más sedas posterofrontales. Palidia ligeramente convergentes en uno o en ambos extremos, cada palidium formado por 11-22 pali .............................. 13 12. Acanthoparia con 7-10 sedas recurvadas. Maxilas con 11-15 dientecillos estriduladores. Cada palidium formado por 15-27 pali cortos. Anchura de la cápsula cefálica 4.1$4.8 \mathrm{~mm}$.......... P. (Phytalus) obsoleta (Blanchard) 12' Acanthoparia con 12-13 sedas recurvadas. Maxilas con 12 dientecillos estriduladores. Cada palidium formado por 18-20 pali cortos. Anchura de cápsula cefálica 4.7 $\mathrm{mm}$................ (Phyllophaga) lenis (Horn) 13. Frente con 2 sedas posterofrontales. Ambos extremos de las palidia convergentes, cada palidium formado por 11-18 pali cortos. Anchura de la cápsula cefálica 6.1- 6.7 $\mathrm{mm}$............. (Phyllophaga) tenuipilis (Bates) 13 ' Frente con 4 sedas posterofrontales. Extremos anteriores de las palidia convergentes, cada palidium formado por 20- 22 pali cortos. Anchura de la cápsula cefálica 4.6$4.8 \mathrm{~mm}$............ (Phyllohaga) brevidens (Bates)

AGRADECIMIENTOS. Se agradece el apoyo del proyecto SIP20144658. El Dr. Roberto Arce Pérez y el Biól. Cesar V. Rojas (INECOL, Xalapa) apoyaron en aspectos técnicos de este trabajo.

\section{LITERATURA CITADA}

Aragón, A. \& Morón, M. A. 2004a. Descripción de las larvas de tres especies de Phyllophaga (Coleoptera: Melolonthidae: Melolonthinae) del Valle de Puebla. Folia Entomológica Mexicana, 43: 295-306.

Aragón, A. \& Morón, M. A. 2004b. Un método de cría para "gallina ciega" rizófagas del género Phyllophaga (Coleoptera: Melolonthidae). Pp. 109-118. In: M. N. Bautista, M. Bravo \& C. Chavarin (Eds). Cría de insectos plaga y organismos benéficos. CONABIO y Colegio de Posgraduados, Montecillos, Texcoco, Estado de México.

Aragón, A., Morón, M. A., López, J. F. \& Cervantes-Peredo, L. M. 2005. Ciclo de vida y conducta de adultos de cinco especies de Phyllophaga Harris, 1827 (Coleoptera: Melolonthinae). Acta Zoológica Mexicana (n.s.), 21: 87-99.

Böving, A. G. 1942. A classification of larvae and adults of the genus Phyllophaga (Coleoptera: Scarabaeidae). Memoirs of the Entomological Society of Washington, 2: 1-95.

Espinosa-Islas, A., Morón, M. A., Sánchez, H., Bautista, N. \& Romero, J. 2005. Complejo gallina ciega (Coleoptera: Melolonthidae) asociado con céspedes en Montecillo, Texcoco, Estado de 
México. Folia Entomológica Mexicana, 44 (2): 95-107.

Hernández-Cruz, J., Morón, M. A., Ruiz-Vega, J., Sánchez-García, J. A., Martínez-Martínez, L. \& Pérez-Pacheco, R. 2014. Bionomía de las especies de Phyllophaga (Coleoptera: Melolonthidae) en Santa Cruz Xoxocotlán, Oaxaca, México. Acta Zoológica Mexicana (n.s.), 30(1): 144-160.

Morón, M. A. 1986. El género Phyllophaga en México. Morfología, distribución y sistemática supraespecifica (Insecta: Coleoptera). Publicación 20. Instituto de Ecología, México.

Morón, M. A. 2003. Diversidad, distribución e importancia de las especies de Phyllophaga Harris en México (Coleoptera: Melolonthidae). Pp. 1-27. In: A. Aragón, M. A. Morón \& A. Marín (Eds.).
Estudios Sobre Coleópteros del Suelo en América. Publicación especial de la Benemérita Universidad Autónoma de Puebla.

Morón, M.A. \& Terrón, R. 1988. Entomología Práctica. Publicación 22. Instituto de Ecología. A.C. México D.F.

Ramírez-Salinas, C., Morón, M. A. \& Castro, A. 2000. Descripción de los estados inmaduros de seis especies de Phyllophaga (Coleoptera: Melolontidae: Melolonthinae) de la región Altos de Chiapas, México. Folia Entomológica Mexicana, 109: 73-106.

Velasco, R. G. J. \& Aguilar, H. 2000. Monografía del municipio de Santa Cruz Xoxocotlán, Centro, Oaxaca. Instituto Politécnico Nacional, Centro Interdisciplinario de Investigación para el Desarrollo Integral Regional. 